\title{
Wall Shear Rates in Taylor Vortex Flow
}

\author{
V. Sobolik ${ }^{1 \dagger}$, T. Jirout ${ }^{2}$, J. Havlica ${ }^{3}$ and M. Kristiawan \\ ${ }^{1}$ University of La Rochelle, Pole Science, LEPTIAB, La Rochelle, 17042, France \\ ${ }^{2}$ Czech Technical University, Faculty of Mechanical Engineering, Prague, 16000, Czech Republic \\ ${ }^{3}$ Institute of Chemical Process Fundamentals AS CR, Prague, 16502, Czech Republic
}

†Corresponding Author Email: vsobolik@univ-lr.fr

(Received January 5, 2009; accepted August 12, 2010)

\begin{abstract}
Wall shear rate and its axial and azimuthal components were evaluated in stable Taylor vortices. The measurements were carried out in a broad interval of Taylor numbers (52-725) and several gap width $\left(R_{1} / R_{2}=0.5-0.8\right)$ by two three-segment electrodiffusion probes and three single probes flush mounted in the wall of the outer fixed cylinder. The axial distribution of wall shear rate components was obtained by sweeping the vortices along the probes using a slow axial flow. The experimental results were verified by CFD simulations. The knowledge of local wall shear rates and its fluctuations is of primordial interest for industrial applications like tangential filtration, membrane reactors and bioreactors containing shear sensitive cells.
\end{abstract}

Keywords: Taylor-Couette flow, Electrodiffusion diagnostics, Tangential filtration, Membrane reactors.

\section{NOMENCLATURE}

$\begin{array}{ll}d & \text { gap width }=R_{2}-R_{1} \\ G & \text { torque } \\ G^{*} & \text { normalized torque }=G / \rho h \Omega^{2} R_{1}{ }^{4} \\ g & \text { normalized torque }=G / \mu \mathrm{h} \Omega R_{1}{ }^{2} \\ h & \text { height of cylinder } \\ R_{I} & \text { radius of inner cylinder } \\ R_{2} & \text { radius of outer cylinder } \\ r, z, \theta & \text { cylindrical coordinates, see Fig. } 4 \\ R e & \text { Reynolds number }=\Omega R_{I} d / v \\ T a & \text { Taylor number }=\Omega R_{l}^{0.5} d^{1.5} / v \\ v & \text { velocity } \\ \text { Greek letters } & \\ \gamma & \text { wall shear rate } \\ \mu & \text { dynamic viscosity } \\ v & \text { kinematic viscosity }\end{array}$

\section{INTRODUCTION}

Vedantam and Joshi (2006) reviewed numerous applications of the Couette-Taylor flow (CTF). Due to its cellular vortex structure, CTF is claimed to be one of the rare flow types combining intense local mixing with a limited axial dispersion (Desmet et al. 1996). Other advantage of CTF is the enhanced heat and mass transfer at the cylinder walls. A vast number of possible applications of the unique reactor performance of CTF covers the field of catalytic and biocatalytic (Baron and Van Capellen 1990), electrochemical (Mizushina 1971; $\mathrm{Gu}$ and Fahidy 1985; Coeuret and Legrand 1981), photochemical (Haim and Pismen 1994) and

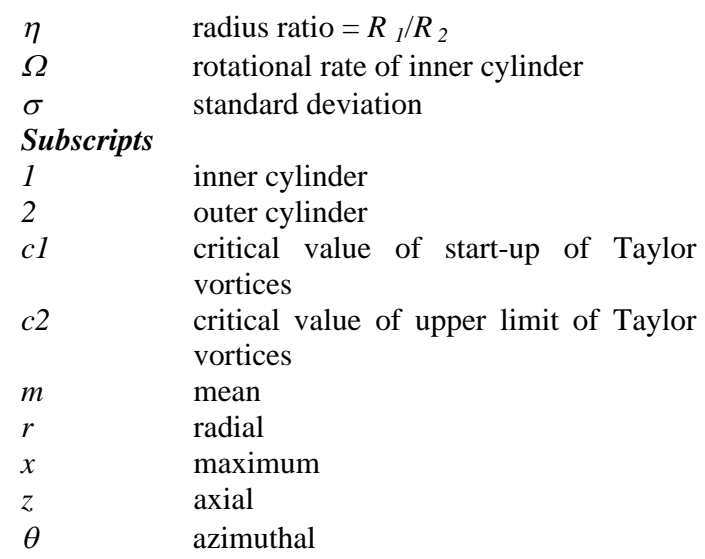

polymerization (Sinevic et al. 1986; Kataoka and Okubo 1995) reactions as well as the field of counter current extraction (Leonard et al. 1981), tangential filtration (Holesovsky and Cooney 1991) and crystallization (Kang et al. 2003).

Many studies concerning flow patterns in CTF have been accomplished, but the knowledge of wall shear rate is mostly qualitative. The wall shear rates are primordial for the applications like membrane filtration (Choi et al. 1999), the reactors with catalyst or enzyme immobilised at a wall (Dutta and Ray 2004) and the bioreactors containing shear sensitive cells (Curran et al. 2004). 
The mean values of wall shear rate were calculated from simple torque measurements (Donnelly and Simon 1960) and linear theories (Eagles 1974). However the local shear rates in CTF are function of the axial coordinate. Their maxima are the most important quantity in the above mentioned applications. The numerical simulations are rather devoted to velocity profiles than to wall shear rates Sengupta et al. (2001). The experimental methods like Laser-Doppler anemometry (Curran and Black 2004) and PIV (Akonur and Lueptow 2003) do not allow measurements in the wall vicinity which is necessary for correct evaluation of wall shear rate. The electrodiffusion diagnostics (ED) is a convenient method for the measurements of this quantity Mizushina (1971). Using three-segment micro-probes the components of wall shear rate can be measured (Wouahbi et al. 2007).

In this work, the axial and azimuthal components of the instantaneous shear rate on the wall of the outer fixed cylinder are measured in a broad interval of rotation rate of the inner cylinder and several gap widths. The axial distribution of wall shear rate components is obtained by sweeping the vortices along the probes using a slow axial flow. The experimental results are confronted with Computational Fluid Dynamics (CFD) simulations. The wall shear stress can be calculated from the wall shear rate via viscosity function.

\section{EXPERIMENTAL}

The experimental apparatus is shown in Fig. 1. It consisted of an outer cylinder 3 made of a Plexiglas tube with an inner radius of $R_{2}=31 \pm 0.2 \mathrm{~mm}$ and an interchangeable inner Plexiglass cylinder 4 . The inner cylinders had a length of $275 \mathrm{~mm}$ and radii of $R_{l}=24.8$, $21.7,18.6$, and 15.5. For calibration of electrodiffusion probes in situ, another cylinder with a radius of 29.5 $\mathrm{mm}$ was used. In this small gap of $1.5 \mathrm{~mm}$, the Couette flow is stable even at higher rotation rates. Thus the probes could be calibrated with well-defined wall shear rates. The corresponding radius ratios, $\eta=R_{1} / R_{2}$, were $0.95,0.8,0.7,0.6$, and 0.5 . The inner cylinder was mounted on the stainless steel shaft 5 which had an upper ball bearing and bottom polyamide sliding bearing. The shaft was driven by a stepping motor with a step of $0.9^{\circ}$ and a gear box with a slow-down ratio of 1:9. There was a plastic clutch between the shaft and gear box which also served as electrical insulation. The revolutions were controlled by a computer directly from the measuring software. Two three-segment electrodiffusion probes 1,2 were embedded in the wall of the outer cylinder. For measurements of the height and drifting velocity of vortices, three simple electrodiffusion probes were flush mounted in the same wall with a vertical distance of $6 \mathrm{~mm}$.

With the aim of obtaining the axial distribution of the velocity gradient components, the Taylor vortices were swept along the probes by a small axial flow. The liquid was circulated by a pump (Fluid Metering Inc.) with a valveless piston head (RH1CKC) having a piston of a diameter of $1 / 4$ '. The maximum volume per stroke was $0.1 \mathrm{~mL}$ and the maximum flow rate was $0.81 \mathrm{~mL} / \mathrm{s}$. The test liquid was pumped from a small tank with a volume of one liter into an inlet tube in the bottom of the apparatus. An outlet tube was mounted $8 \mathrm{~mm}$ below the cover. It was connected with the tank by a hose.

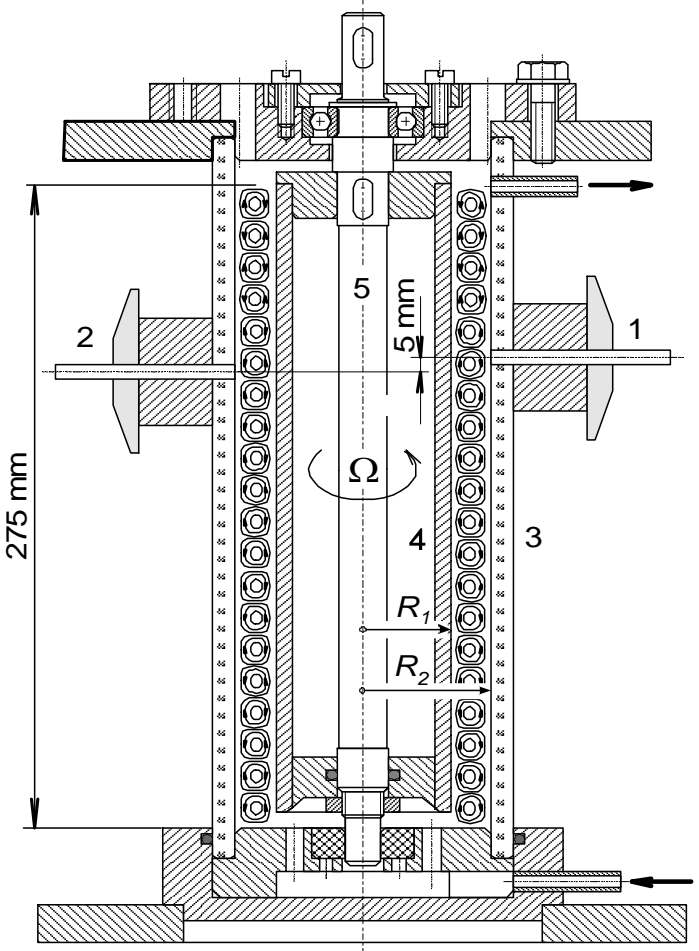

Fig. 1. Experimental set-up. 1, 2 three-segment electrodiffusion probes, 3 outer cylinder, 4 inner cylinder, 5 shaft.

The measurement of wall shear rate by means of the limiting diffusion current is a well-known technique. A two-electrode cell consisting of a small working electrode and a large auxiliary electrode, a solution containing depolarizer and supporting electrolyte in excess are used for measuring the limiting diffusion current. The applied voltage must have a value at which only the active species react on the working electrode probe. As the reaction is rapid, the concentration of the active species is negligibly small at the working electrode. The test liquid used in the experiments was a $25 \mathrm{~mol} / \mathrm{m}^{3}$ equimolar potassium hexacyanoferrates (III) and (IV) aqueous solution with $1.5 \%$ b.w. $\mathrm{K}_{2} \mathrm{SO}_{4}$ as supporting electrolyte. The electrolyte had a viscosity of $1.05 \mathrm{mPa}$ s at $21.3^{\circ} \mathrm{C}$ and a density of $1010 \mathrm{~kg} / \mathrm{m}^{3}$. The flow was visualized by addition of $2 \%$ of rheoscopic liquid AQ-1000 (Kalliroscope Corp., U.S.A.). The rheoscopic liquid contains small laminae reflecting light in dependence on their orientation imposed by the flow direction.

The three-segment probes were made in house. Three platinum wires with a diameter of $0.5 \mathrm{~mm}$ were pulled simultaneously through a wire-drawing die, starting with a diameter of $1 \mathrm{~mm}$ and finishing with $0.5 \mathrm{~mm}$. The wires were then insulated by a layer of polymeric paint and glued together with epoxy resin. After soldering the connecting cables, the wires were cemented with the resin into a stainless steel tube with a tip diameter of $3 \mathrm{~mm}$. The top view of a probe is shown in Fig. 2. The small ring between the probe and the tube is a remainder of burr after machining of the tube face. 


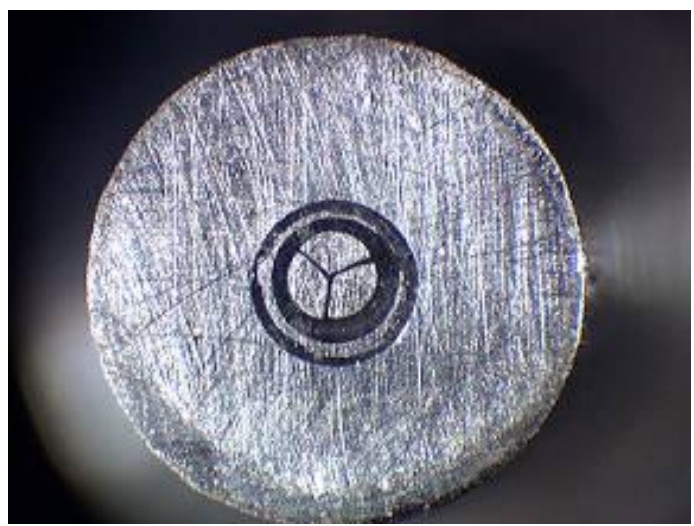

Fig. 2. Top view of three-segment probe with a diameter of $0.5 \mathrm{~mm}$ embedded in a tube with $3 \mathrm{~mm}$ outside diameter.

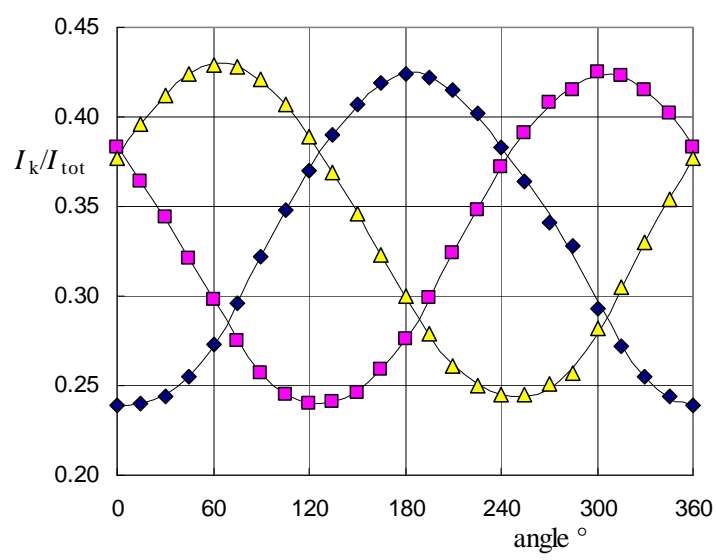

Fig. 3. Dependence of segment currents normalized by their sum on flow angle.

For the evaluation of flow direction, we make use of the non-linear dependence of the limiting current density on the distance from the forward electrode edge, $i(x) \sim x^{-1 / 3}$ (Lévêque 1928). The forward segment has higher current than the segment that lies in his shade. Hence, the absolute value of wall shear rate is calculated from the sum of the limiting diffusion currents passing through the segments and the flow direction (components of wall shear rate) from the ratios of these currents (Wein and Sobolik 1987).

The three-segment probe was calibrated in the apparatus with a larger inner cylinder $(\eta=0.95)$ where the laminar Couette flow with wall shear rates in the interval $\left(5 ; 80 \mathrm{~s}^{-1}\right)$ could be adjusted $(2.5-40 \mathrm{rpm})$. The sum of segment currents were measured as a function of $\gamma$. We assumed that the limiting diffusion current is power law function of wall shear rate $\left(I=K \gamma^{\mathrm{n}}\right)$. For decomposition of the wall shear rate into axial and azimuthal components, directional calibration at $\gamma=20 \mathrm{~s}^{-1}$ was curried up. The probe was turned by $15^{\circ}$ steps and the currents of three segments were measured in the range $\left(0 ; 360^{\circ}\right)$. The dependences of the currents normalized by the sum of currents on flow angle are called directional characteristics, see Fig. 3. Every flow angle corresponds to a unique combination of three currents.

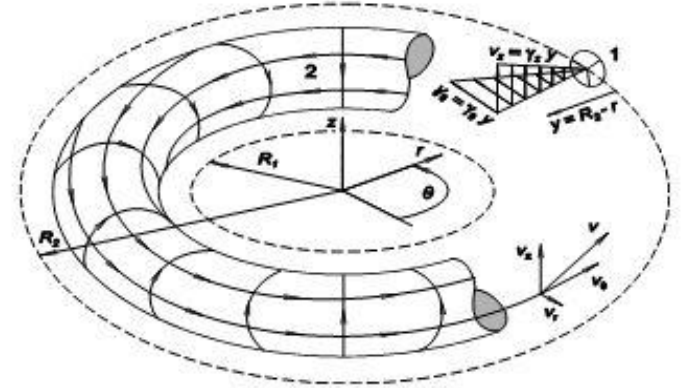

Fig. 4. Schematized Taylor vortex: 1 probe, 2 Taylor vortex tube, $\gamma_{z}$ axial component of wall shear rate, $\gamma_{\theta}$ azimuthal component of wall shear rate.

When the Taylor number reaches a critical value (first transition), laminar Couette flow is no longer stable and disturbances appear which ultimately take the form of cellular, toroidal vortices, regularly spaced along the axis $\mathrm{z}$. The cylindrical coordinates $r, \theta, z$, the corresponding velocity components $v_{r}, v_{. \theta}, v_{z}$, and a "stream tube" of a Taylor vortex are shown in Fig. 4. The velocity field at the probe 1 are shown in a magnified view. The wall shear rate components on the inner and outer cylinder are defined by

$$
\begin{aligned}
& \gamma_{z 1}(z)=\left.\frac{\partial v_{z}(z)}{\partial r}\right|_{r=R 1}, \quad \gamma_{z 2}(z)=-\left.\frac{\partial v_{z}(z)}{\partial r}\right|_{r=R 2} \\
& \gamma_{\theta 1}(z)=-\left.\frac{\partial v_{\theta}(z)}{\partial r}\right|_{r=R 1}, \quad \gamma_{\theta 2}(z)=-\left.\frac{\partial v_{\theta}(z)}{\partial r}\right|_{r=R 2}
\end{aligned}
$$

Mean value of the azimuthal component is defined by

$\gamma_{\theta i, m}=\frac{1}{h} \int_{0}^{h} \gamma_{\theta i} d z$

In our experiments, the mean value of axial components $\gamma_{\text {zim }}$ over vortex pair is equal to the wall share rate of the axial flow $\gamma_{\mathrm{ax}}$.

If the rotational rate is further increased, azimuthal waves are superposed on the toroidal vortices. In this work, the wall shear rate components were measured in regular Taylor vortices at several rotation rates. The experiments were carried out as follows. The gap between the cylinders was filled with the solution up to the outlet tube and the pump was stopped. Critical Taylor number of the first transition was found by increasing gradually the rotation rate of the cylinder until the start-up of vortices. This state was indicated by a non zero axial component of wall shear rate and also by pattern visualisation. Then the velocity was adjusted on selected value. Once the Taylor vortices were steady and fully developed, a slow axial flow of $0.12 \mathrm{~mL} / \mathrm{s}$ was set up.

\section{NUMERICAL}

Hydrodynamics of stable Taylor vortices was also investigated by CFD. The simulations were done with the commercial solver Fluent 6.2. The calculations were carried out in a 2D axisymmetric domain. The gap widths $d$ between two cylinders were the same as in the experimental device. The height of vortices was 
imposed to be equal to the gap width, see Fig. 5.

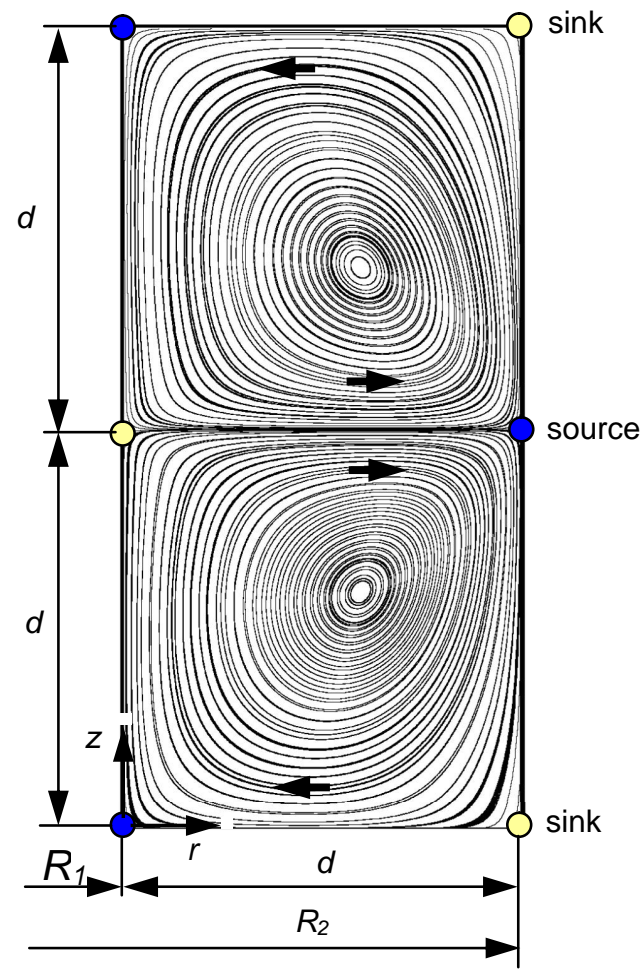

Fig. 5. Streamlines in $r-z$ plane of two vortices for $\eta=0.5, T a=725, \Omega=16.4$.

The domain included two pairs of vortices. The grids were uniform having between 60000 and 80000 cells. Steady-state, laminar and incompressible flow was considered. The conservation equations of mass and momentum were written in a cylindrical coordinate system for 2D swirling flow. The pressure-velocity coupling was obtained using the SIMPLEC algorithm. Discretization scheme was used for momentum ThirdOrder MUSCL and for pressure Body Force Weighted. Symmetry boundary conditions were imposed on the separation planes $z=\mathrm{k} d$ ( $\mathrm{k}=0$ and 4$)$. It means, that there is no convective flux across the symmetry plane and the axial velocity component $v_{z}$ is zero. No-slip boundary conditions were applied on the walls of the cylinders: the velocity components $v_{z}\left(R_{l}, R_{2}\right), v_{r}\left(R_{l}\right.$, $\left.R_{2}\right)$ and $v_{\theta}\left(R_{2}\right)$ were zero and $v_{\theta}\left(R_{l}\right)$ equal to $\Omega R_{l}$.

\section{RESUlTS AND DISCUSSION}

The measured data on $T a_{c l}$ coincided well with the literature data. In the interval $\eta(0.5 ; 1)$, they were fitted by the equation

$T a_{c 1}=36.2(\eta-0.256)^{-0.445}$

with a standard deviation of 0.24. This equation approaches the data better than that of Choi et al. (1999) with the exception of $\eta \rightarrow 1$. The regime of Taylor vortices separated by planes stays until the second transition where azimuthal waves are superposed on these vortices. The literature information about $T a_{c 2}$ is scare. In our experiments, the critical $T a_{c 2}$ for $\eta=0.8$ and 0.7 had reproducible values 80.5 and 553 , respectively. In the larger gaps, the free surface had a disturbing effect on the stability of Taylor vortices; hence it was eliminated by insertion of a ring fixed on the outer cylinder. In these experiments, it was necessary to increase the rotation rate by small steps and wait about 10 minutes for a full flow development. Then values of $T a_{c 2} 789$ and 1057 were found for $\eta=0.6$ and 0.5 , respectively.

The second transition, i.e. Taylor vortices with azimuthal waves, depends strongly on the aspect ratio (number of vortex pairs) (Cole 1976). For $\eta=0.8703$, Edwards et al. (1991) found $T a_{c 2}=234$ and 52 for 3 and 17 vortex pairs, respectively. This effect can be explained by the Ekman vortices which are formed on the motionless annular boundary. These vortices decrease the critical number of the first transition and increase the critical number of the second transition. It means that the Ekman vortices damp the wave formation on the Taylor vortices. Cole (1976) stated that $T a_{c 2}$ does not depend on aspect ratio for the values exceeding 40. In our experiments, this criterion was satisfied only for $\eta=0.8$.

The measured values of wall shear rate components were fitted by Fourier series of sixth order. The agreement between measured and numerically simulated data was better than $10 \%$. Only the lowest wall shear rates about $1 \mathrm{~s}^{-1}$ were measured with an error of $20 \%$ due to the effect of longitudinal diffusion. The experimental height of vortices was equal to the gap width within $10 \%$. Examples of the data obtained in the geometries $\eta=0.8$ and 0.6 are shown in Fig. 6 .
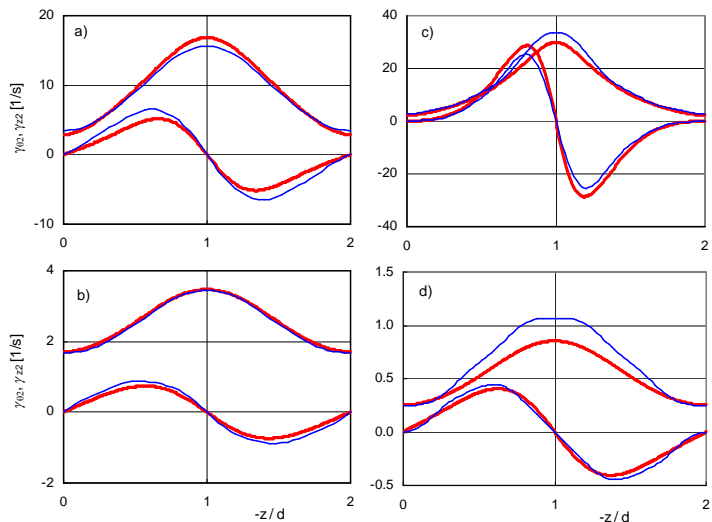

Fig. 6. Comparison of measured (thin line) and simulated (thick line) components of wall shear rate for $\eta=0.8$ : a) $T a=102.5$; b) $T a=51.5$ and for $\eta=0.6$ : c) $T a=591 ;$ d) $T a=78.3$

Wall shear rate and its components are shown in Fig. 7 as a function of axial distance. The axial component has a mean value which is practically equal to zero. For $z / d=0,2$ and 4 , there are sinks (rear critical point) on the outer wall and sources (forward critical point) at inner wall (see also Fig. 5). For $z / d=1$ and 3, sources are at the outer wall and sinks on the inner wall. At the sinks and sources, the axial component takes zero value, whereas the azimuthal component exhibits extremes. The distributions have almost sinusoidal form in a narrow gap $(\eta=0.8)$ at a low Taylor number (Fig. 7a, b). There is not much difference between the wall shear rates at the inner and outer wall. 

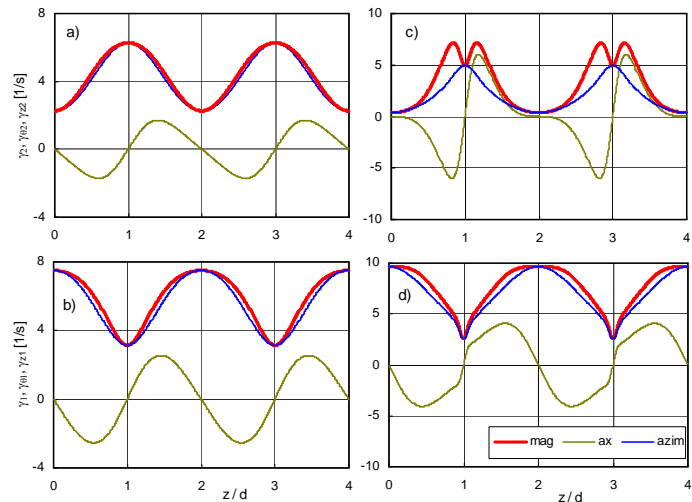

Fig. 7. Wall shear rate and its components. mag: $\gamma$, ax: $\gamma_{z}$, azim $\gamma_{\theta}$

a: outer wall, b: inner wall, $\eta=0.8, T a=52.2, \Omega=3.69$. c: outer wall, d: inner wall, $\eta=0.5, T a=725, \Omega=16.4$.

The ratio of the corresponding values at the inner and outer walls is approximately the same as in the laminar Couette flow, i.e. $\gamma_{1} / \gamma_{2}=\left(1+\eta^{2}\right) / 2 \eta^{2}$. In a large gap at high Taylor number (Fig. 7c, d) a jet flow in the source at the outer wall manifest itself by a steep variation of the axial component. This fact causes a local minimum at the source and two maxima of wall shear rate near the source (camel shape). The maximum of axial component can be superior to the maximum of azimuthal component. Jet flow does not exist at the inner wall. At this wall, the variation of axial velocity is steeper at the sink than at source. Due to low values of $\gamma_{z l}$ at the inner wall, the wall shear rate almost coincides with its azimuthal component for all radius ratio investigated.

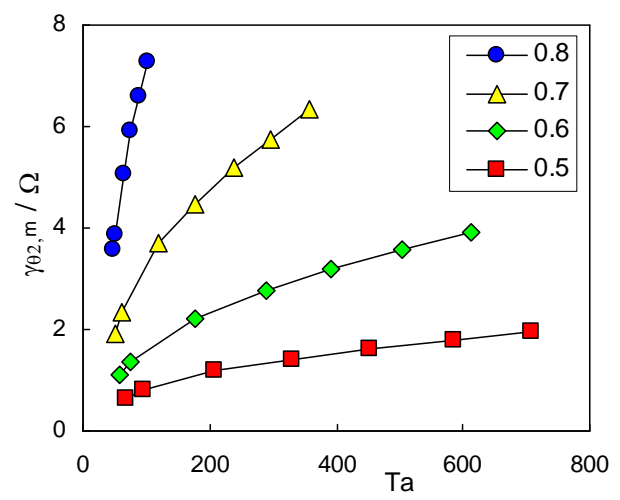

Fig. 8. Normalized values of mean azimuthal wall shear rate component on the outer wall as a function of Taylor number. Radius ratio $\eta$ stands for curve parameter.

The torque on a cylinder can be expressed by means of the mean value of azimuthal wall shear rate component on the outer cylinder normalized by $\Omega$

$\frac{\gamma_{\theta 2, m}}{\Omega}=\frac{G}{2 \pi R_{2}^{2} h \mu \Omega}$

Donnelly and Simon (1960) and Fasel and Booz (1984) introduced dimensionless torque $G^{*}$ and $g$, respectively. There are the following relations among $\gamma_{\theta 2, m}, G^{*}$ and $g$
Table 1 Coefficients of equation 7.

\begin{tabular}{ccccccc}
\hline$\eta$ & $T a_{\mathrm{c} 1}$ & $a$ & $b$ & $c$ & $\sigma$ & $T a_{\mathrm{c} 2}$ \\
\hline 0.8 & 47.5 & -4990 & 1.260 & 0.397 & 0.018 & 80.5 \\
0.7 & 51.9 & -1760 & 0.426 & 0.462 & 0.016 & 553 \\
0.6 & 58.2 & -680 & 0.222 & 0.449 & 0.007 & 789 \\
0.5 & 68.2 & -126 & 0.128 & 0.418 & 0.004 & 1057 \\
\hline
\end{tabular}

$\frac{\gamma_{\theta 2, m}}{\Omega}=G \frac{R_{1}^{2} \rho \Omega}{2 \pi \eta^{2} \mu}=g \frac{\eta^{2}}{2 \pi}$

Normalized mean azimuthal components of wall shear rates are shown in Fig. 8 as a function of Ta with $\eta$ as parameter. These values were fitted by the relation

$\frac{\gamma_{\theta 2, m}}{\Omega}=a T a^{-2}+b T a^{c}$.

This relation is similar to that of Donnelly and Simon (1960). For comparison of our exponents -2 and $c$ with those found in the literature, unity must be added to our values. It is due to the normalisation by $\Omega$. In the following discussion, the coefficients correspond to our normalisation. Stuart (1958) calculated the torque on cylinder by an energy-balance method for the limiting case of a small gap, $\eta \rightarrow 1$. His result is expressed by Eq. (7) with $c=0$. Davey (1962) used a rigorous perturbation expansion for solving the velocity of Taylor vortices under the assumption of a small amplitude. According to Davey (1962) the exponent $c=$ 0 , but the coefficients $a$ and $b$ are different from that of Stuart (1958). Donnelly and Simon (1960) fitted several sets of torque measurements and found $c$ in the interval $(0.33,0.5)$. Batchelor assumed that the vortex flow consists of inviscid cores surrounded by boundary layers and found that $c=0.5$ (appendix to Donnelly and Simon 1960). Such a flow occurs at high Taylor numbers where the first term in Eq. (7) equals zero. The coefficients $a, b, c$, obtained from our numerical simulations and measurements, are given in Table 1 together with $T a_{c}$ and standard deviations. The exponent $c$ coincides well with the value given by Donnelly and Simons (1960).

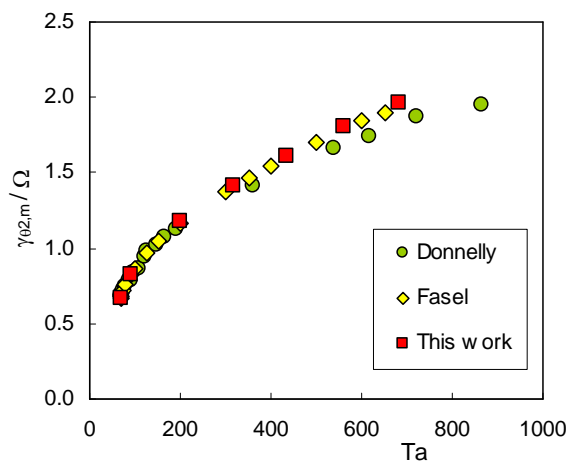

Fig. 9. Comparison of mean azimuthal wall shear rates for $\eta=0.5$ 
Table 2 Comparison of mean azimuthal component of wall shear rate with mean value of wall shear rate.

\begin{tabular}{cccccc}
\hline$\eta$ & Ta & $\gamma_{\theta 1, \mathrm{~m}}$ & $\gamma_{1, \mathrm{~m}}$ & $\gamma_{\theta 2, \mathrm{~m}}$ & $\gamma_{2, \mathrm{~m}}$ \\
\hline 0.8 & 52.2 & 5.56 & 5.85 & 4.20 & 4.36 \\
0.8 & 105 & 10.69 & 11.45 & 7.50 & 8.22 \\
0.5 & 97.2 & 2.42 & 3.60 & 0.85 & 1.03 \\
0.5 & 725 & 7.01 & 7.68 & 2.00 & 3.13 \\
\hline
\end{tabular}

The normalized azimuthal wall shear rate components calculated from measured torque by Donnelly and Simon (1960), simulated numerically by Fasel and Booz (1984) and measured and simulated in this work are compared in Fig. 9. The agreement is rather good until $\mathrm{Ta}=400$. For higher values of $\mathrm{Ta}$, the data of Donnelly and Simon (1960) lay below the data of Fasel and Booz (1984) and the data obtained in this work.

The normalized maximal values of azimuthal components are shown in Fig. 10 as a function of normalized Taylor number. These values are almost independent of $\eta$. The dependence of normalized maximal values of axial components on $\eta$ is more important (see Fig. 11).

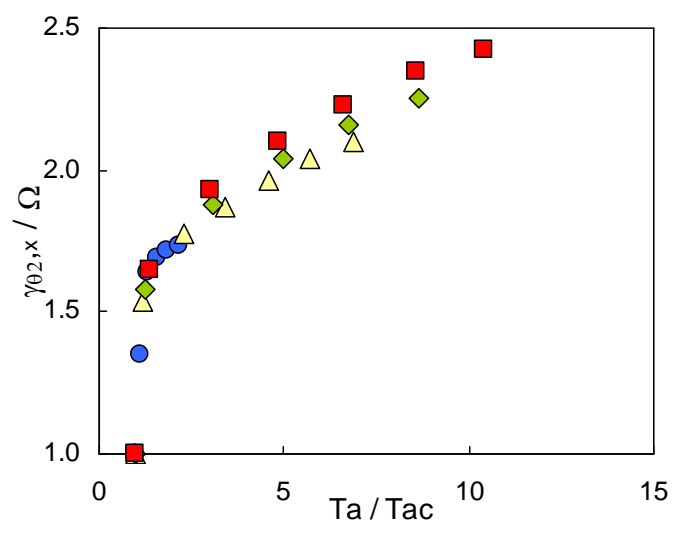

Fig. 10. Maximum values of azimuthal component of wall shear rate on the outer wall. See Fig. 8 for symbols.

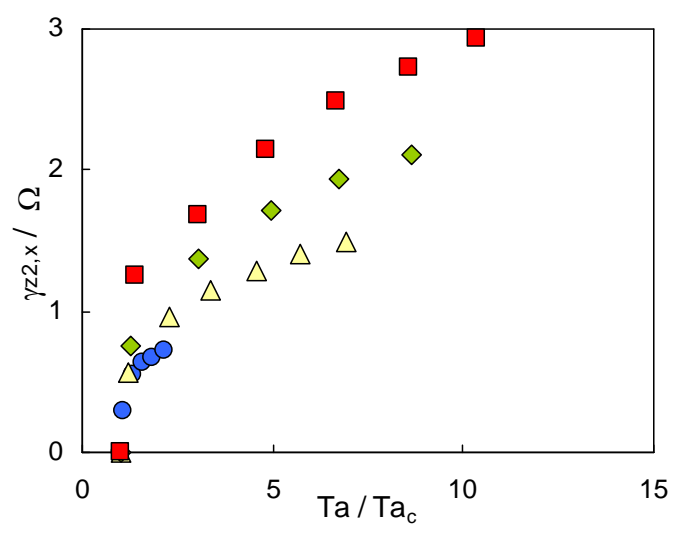

Fig. 11. Maximum values of axial component of wall shear rate on the outer wall. See Fig. 8 for symbols.
It should be remembered that $\gamma_{\theta 1, m}$ and $\gamma_{\theta 2, \mathrm{~m}}$ which can be calculated from measured torque are not equal to the mean values of wall shear rate $\gamma_{1, m}$ and $\gamma_{2}, m$, respectively. It is due to the axial component which can achieved high values. These values are compared in Table 2 for different $T a$ and $\eta$. The difference makes more than $50 \%$ for the shear rates at the outer wall at $\mathrm{Ta}$ $=725$ and $\eta=0.5$.

Gaucher et al. (2002) studied the wall suction effect on the wall shear rate at a plane ultrafiltration surface. They found that the wall shear rates of the order $10^{4} \mathrm{~s}^{-1}$ were doubled using a small suction (ratio of the wall $R e$ to the channel $R e$ of the order $10^{-4}$ ). It will be interesting to study the effect of the wall suction on the wall shear rate in Taylor-Couette flow.

\section{Conclusions}

The mean values and maxima of wall shear rate and its components in regular Taylor vortex flow were found for the radius ratios $0.8,0.7,0.6$ and 0.5 . The Taylor or Reynolds number is the only criterion which characterizes the flow. At a given Taylor number, the value of shear rate or its component normalized by angular velocity of the inner cylinder is unique. It allows us to evaluate shear rate for different fluid viscosity. This conclusion can be generalized for velocity fields. A local minimum at the source and two maxima of wall shear rate near the source (camel shape) at the outer wall start to appear at $T a=120$ in $\eta=0.7$. This effect is more pronounced with decreasing $\eta$ and increasing $T a$. In larger gaps the mean value of wall shear rate calculated from measured torque are lower than its true value. The knowledge of wall shear rate components and their distribution is helpful for conception of apparatus based on Taylor-Couette flow.

\section{ACKNOWLEDGEMENTS}

This work was supported by the "Agence National de la Recherche", France, by the grant number ANR-08BLAN-0184-01.

\section{REFERENCES}

Akonur, A. and R.M. Lueptow (2003). Threedimensional velocity field for wavy TaylorCouette flow. Physics of Fluids 15, 947-960.

Baron, V. and P. Van Capellen (1990). A novel Bioreactor for Immobilised Cell Systems, in Physiology of Immobilized Cells. Elsevier, Amsterdam.

Choi, C.K., J.Y. Park, W.C. Park and J.J. Kim (1999). A study on dynamic separation of silica slurry using a rotating membrane filter: 2 . Modelling of cake formation. J. Membrane Sci. 157, 177-187.

Cole, J.A. (1976). Taylor-vortex instability and annulus-length effects. J. Fluid Mech. 75, 1-15.

Coeuret, F. and J. Legrand (1981). Mass transfer at the electrodes of concentric cylindrical reactors 
combining axial flow and rotation of the inner cylinder. Electrochim. Acta 26, 865-872.

Curran, S.J., A. Richard and R.A. Black (2004). Quantitative experimental study of shear stresses and mixing in progressive flow regimes within annular-flow bioreactors. Chem. Eng. Sci. 59, 5859-5868.

Davey, A. (1962). The growth of Taylor vortices in flow between rotating cylinders. J. Fluid Mech. 14, 336-368.

Desmet, G., H. Verelst and G.V. Baron (1996). Local and global dispersion effects in Couette-Taylor flow - II Quantitative measurements and discussion of the reactor performance. Chem. Eng. Sci. 51, 1299-1309.

Donnelly, R.J. and N.J. Simon (1960). An empirical torque relation for supercritical flow between rotating cylinders. J. Fluid Mech. 7, 401-418.

Dutta, P.K. and A.K. Ray (2004). Experimental investigation of Taylor vortex photocatalytic reactor for water purification. Chem. Eng. Sci. 59, 5249-5259.

Eagles, P.M. (1974). On the torque of wavy vortices. $J$. Fluid Mech. 62, 1-9.

Edwards, W.S., S.R. Beane and S. Varma (1991). Onset of wavy vortices in the finite-length GouetteTaylor problem. Phys. Fluids A 3(6).

Fasel, H. and O. Booz (1984). Numerical investigation of supercritical Taylor-vortex flow for a wide gap. J. Fluid Mech. 138, 21-52.

Gaucher C., P. Jaand ouen, P. Legentilhomme and J. Comiti (2002). Suction effect on the shear stress at a plane ultrefiltration ceramic membrane surface. Separation Sci. Technol. 37, 2251-2270.

Gu, Z.H. and T.Z. Fahidy (1985). Mass transport in the Taylor-vortex regime of rotating flow. Chem. Eng. Sci. 40, 1145-1153.

Haim, D. and L.M. Pismen (1994). Performance of a photo-chemical reactor in the regime of TaylorGoertler vortical flow. Chem. Eng. Sci. 49, 11191129.

Hanratty, T.J. and J.A. Campbell (1983). Measurement of Wall Shear Stress. In: Goldstein RJ (ed.) Fluid Mechanics Measurements, Hemisphere Publishing Corporation, Washington.

Holechovsky, U.B. and C.L. Cooney (1991). Quantitative description of ultrafiltration in a rotating filtration device. A.I.Ch.E.J. 37, 12191227.
Kang, S.H., S.G. Lee, W.M. Jung, M.C. Kim, W.S. Kim, C.K. Choi and R.S. Feigelson (2003). Effect of Taylor vortices on calcium carbonate crystallization by gas-liquid reaction. J. Crystal Growth 254, 196-205.

Kataoka, K. and M. Okubo (1995). Emulsion polymerization of styrene in a continuous Taylor vortex flow reactor. Chem. Eng. Sci. 50, 14091416.

Leonard, R.A., G.J. Bernstein, R.H. Pelto and A.A. Ziegler (1981). Liquid--liquid dispersion in turbulent Couette flow. A.I.Ch.E.J. 27, 495-503.

Lévêque, M.A. (1928). Les lois de la transmission de la chaleur par convection. Ann. Mines 13, 201-239.

Mizushina, T. (1971). The Electrochemical Method in Transport Phenomena, Advances in Heat Transfer. Academic Press, New York.

Sengupta, T.K., M.F. Kabir and A.K. Ray (2001). A Taylor photocatalytic reactor for water purification. Ind. Eng. Chem. Res. 40, 5268-5281.

Sinevic, V., R. Kuboi and A.W. Nienow (1986). Power numbers, Taylor numbers and Taylor vortices in viscous Newtonian and non-Newtonian fluids. Chem. Eng. Sci. 41, 2915-2923.

Sobolik, V., J. Legrand, A. Latifi and O. Wein (2007). Calibration of a segmented friction probe flush mounted in a sphere. Measuring Sci. Technol. 18, 2704-2711.

Stuart, J.T. (1958). On the non-linear mechanics of hydrodynamic stability. J. Fluid Mech. 4, 1.

Vedantam, S. and J.B. Joshi (2006). Annular centrifugal contactors - A Review 2006. Chem. Eng. Research and Design 84, 522-542.

Wein, O. and V. Sobolik (1987). Theory of direction sensitive probes for electrodiffusion measurement of wall velocity gradients. Collect. Czech. Chem. Commun. 52, 2169-2180.

Wouahbi, F., K. Allaf and V. Sobolik (2007). Electrochemical analysis of Taylor vortices. J. Appl. Electrochem. 37, 57-62, 10.1007/s10800006-9211-2. 